\title{
Direct Observation of Resonant Tunneling Dynamics in High Magnetic Fields
}

\author{
A. P. Heberle ${ }^{1}$ M. Oestreich,${ }^{1}$ S. Haacke,${ }^{2}$ W. W. Rühle,${ }^{1}$ J. C. Maan, ${ }^{3}$ and K. Köhler ${ }^{4}$ \\ ${ }^{1}$ Max-Planck-Institut für Festkörperforschung, Heisenbergstraße 1, D-70506 Stuttgart, Germany \\ ${ }^{2}$ Grenoble High Magnetic Field Laboratory, Max-Planck-Institut für Festkörperforschung-Centre Nationale de la Recherche \\ Scientifique, B.P.166, F-38042 Grenoble Cédex 9, France \\ ${ }^{3}$ High Field Magnet Laboratory and Research Institute for Materials, University of Nijmegen, 6525 ED Nijmegen, \\ The Netherlands \\ ${ }^{4}$ Fraunhofer-Institut für Angewandte Festkörperphysik, Tullastraße 72, D-79108 Freiburg, Germany
}

(Received 2 August 1993)

\begin{abstract}
Magnetotunneling of electrons in an asymmetric double quantum well is investigated by timeresolved luminescence spectroscopy. The tunneling time decreases strongly when the bottom of the lowest electronic subband of the narrow well is brought to intersect the lowest wide well subband at nonzero wave vector by a magnetic field perpendicular to the growth direction. This decrease is particularly pronounced when longitudinal optical phonon emission is possible. Transfer becomes then as fast as in electrically tuned resonances in zero magnetic field.

PACS numbers: $73.40 . \mathrm{Gk}, 72.20 . \mathrm{My}, 78.47 .+\mathrm{p}$
\end{abstract}

The understanding of the quantum mechanisms for electron tunneling in semiconductor structures is a key issue for the development of novel electronic devices $[1,2]$. The rates of tunneling transport determine operational speed, noise, and energy consumption. Switching can be achieved by exploiting the huge enhancement of tunneling rates at energy resonances. The tunneling processes at resonance are strongly influenced by other basic electronic processes, such as scattering by defects, i.e., impurities and interface roughness or by interaction with phonons [3,4]. The reduction of tunneling time in resonance is a general principle of physics; however, this view is simplifying in two-dimensional systems if the in-plane dispersion of the carriers is neglected.

A magnetic field modifies the tunneling dynamics according to the orientation of the field with respect to the direction of electron motion: a field $B_{\|}$(i.e., along the growth direction) leads to tunneling between quantized Landau levels [5], while a perpendicular field $B_{\perp}$ shifts the resonances because of conservation of the transverse canonical momentum [6], allowing electrons to be injected resonantly at finite energies and finite in-plane wave vectors in the target well. The dynamics of tunneling is in this case sensitive to the in-plane dispersion. In a perpendicular field only transport experiments have thus far been done [7-9], but no information has been obtained on the detailed dynamics. Time-resolved optical experiments, on the other hand, yield such information [10-12] but have to date not been performed in high magnetic fields; i.e., little information on the influence of in-plane motion on the physics of tunneling could be deduced.

This paper describes the first direct determination of resonant and nonresonant magnetotunneling times of electrons from a narrow quantum well $\left(\mathrm{QW} \mathrm{W}_{n}\right)$ to a wide quantum well $(\mathrm{QW} w)$ through a single potential barrier in an asymmetric double quantum well (ADQW) by time-resolved picosecond photoluminescence spectroscopy. Only the combination of external electric and magnetic fields with time-resolved optical experiments allows one to get novel information about how the in-plane motion of the carriers influences the tunneling transfer process. This process actually changes completely from a defect assisted transfer to an intrinsic, optical phonon assisted transfer if the resonance occurs between equal or different in-plane motion of the carriers. The transfer times are in both cases almost equal.

The inset of Fig. 1 shows the conduction (CB) and valence band (VB) of the ADQW with the lowest electron and hole subband edges. The arrows show the channels for recombination and electronic tunneling [13]. The tunneling time out of a quantum well is defined as the inverse rate of electrons leaving $\mathrm{QW}_{n}$ through the barrier. Such an unambiguous definition of a tunneling time often does not exist in transport experiments [14]. We demonstrate that the tunneling time strongly decreases if the bottom of the lowest electronic subband $|n 1\rangle$ of $\mathrm{QW}_{n}$ is tuned

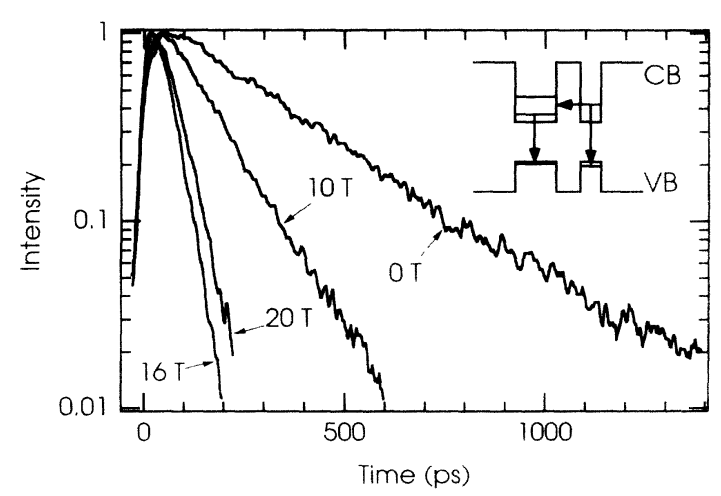

FIG. 1. Luminescence decay of $\mathrm{QW} n$ for different magnetic fields perpendicular to the growth direction at a sample temperature of $80 \mathrm{~K}$. Inset: Conduction $(\mathrm{CB})$ and valence band (VB) of an ADQW in the growth direction with the lowest electron and hole levels. The arrows indicate recombination and electronic tunneling. 
in resonance with the lowest subband $|w 1\rangle$ of $\mathrm{QW}_{w}$ by a strong magnetic field $B_{\perp}$.

The experiments are based on a sample grown by molecular beam epitaxy on an $n^{+}$-doped GaAs:Si substrate with layers in the following sequence: buffer structure, $100 \mathrm{~nm} \mathrm{Al}_{0.35} \mathrm{Ga}_{0.65} \mathrm{As}, 10$ times the ADQW structures separated by $20 \mathrm{~nm} \mathrm{Al} \mathrm{Al}_{0.35} \mathrm{Ga}_{0.65} \mathrm{As}, 100 \mathrm{~nm}$ $\mathrm{Al}_{0.35} \mathrm{Ga}_{0.65} \mathrm{As}$, and a $5 \mathrm{~nm} \mathrm{GaAs}$ layer on top. The ADQW structures consist of a $10 \mathrm{~nm}$ wide GaAs QW, a $6 \mathrm{~nm} \mathrm{Al}_{0.35} \mathrm{Ga}_{0.65}$ As barrier, and a $5 \mathrm{~nm}$ narrow $\mathrm{GaAs}$ $\mathrm{QW}$. For experiments with electric field $\mathrm{Ohmic} \mathrm{Au}-\mathrm{Ge} / \mathrm{Ni}$ contacts are alloyed into the substrate and semitransparent $5 \mathrm{~nm}$ thick Ni-Cr Schottky contacts of $1 \mathrm{~mm}$ diameter are evaporated on top. The $\mathrm{Al}_{0.35} \mathrm{Ga}_{0.65} \mathrm{As}$ barriers permit both polarities of the electric field at low temperatures. The electric fields are calibrated from the shift of the $\mathrm{QW}_{w}$ luminescence to lower energy caused by the quantum confined Stark effect.

The samples are excited with pulses from a tunable Styryl 8 dye laser, which is synchronously pumped by a frequency doubled Nd:YAG laser at a repetition rate of 76 $\mathrm{MHz}$. The carrier excitation density is below $10^{10} \mathrm{~cm}^{-2}$. The excitation energy is tuned just above the ground state of $\mathrm{QW}_{n}$. The luminescence decay times of the $A D Q W$ are not found to be sensitive to the excitation energy. The photoluminescence is dispersed by a $0.32 \mathrm{~m}$ spectrometer and detected by a two-dimensional streak camera with a spectral and temporal resolution of $0.5 \mathrm{~nm}$ and $15 \mathrm{ps,}$, respectively.

Figure 1 shows the influence of a magnetic field $B_{\perp}$ on the luminescence decay of $\mathrm{QW}_{n}$ for a sample temperature of $80 \mathrm{~K}$. The magnetic field strongly reduces the luminescence decay time $\tau_{n}$ of $\mathrm{QW}_{n}$ with a pronounced minimum around $16 \mathrm{~T}$. A similar decrease is not observed in the luminescence decay time $\tau_{w}$ of $\mathrm{QW}_{w}$. The luminescence decay rate $\tau_{n}^{-1}$ of $\mathrm{QW} n$ is the sum of the recombination rate $\tau_{R}^{-1}$ and the electronic tunneling rate $\tau_{T}^{-1}$ from $\mathrm{QW}_{n}$ to $\mathrm{QW}_{w}: \tau_{n}^{-1}=\tau_{R}^{-1}+\tau_{T}^{-1}$. The luminescence decay reflects the influence of the magnetic field on tunneling. Figure 2 shows the dependence of $\tau_{n}$ on the magnetic field for sample temperatures of $2 \mathrm{~K}$ and $80 \mathrm{~K}$. The different decay times at low magnetic field are due to the temperature dependent recombination rate [15] which slightly influences $\tau_{n}$ at a temperature of $2 \mathrm{~K}$. The decay times indicate a tunneling resonance around $16 \mathrm{~T}$ where $\tau_{n}$ is 1 order of magnitude smaller than for $0 \mathrm{~T}$. The width of the resonance increases with temperature. No significant influence of the magnetic field on $\tau_{n}$ is observed in a magnetic field $B_{\|}$in growth direction.

The main effect of the magnetic field $B_{\perp}$ in the $x$ direction is to shift the dispersion of $|n 1\rangle$ against that of $|w 1\rangle$ in the $y$ direction by an amount $\Delta k_{y}=e \Delta z B_{x} / \hbar$, since for thin wells the diamagnetic shift of the lowest subband edges with energy $E_{n 1}$ and $E_{w 1}$ can be neglected [16]. The distance $\Delta z=\langle z\rangle_{w 1}-\langle z\rangle_{n 1}$ which the electrons tunnel is the difference of the expectation values

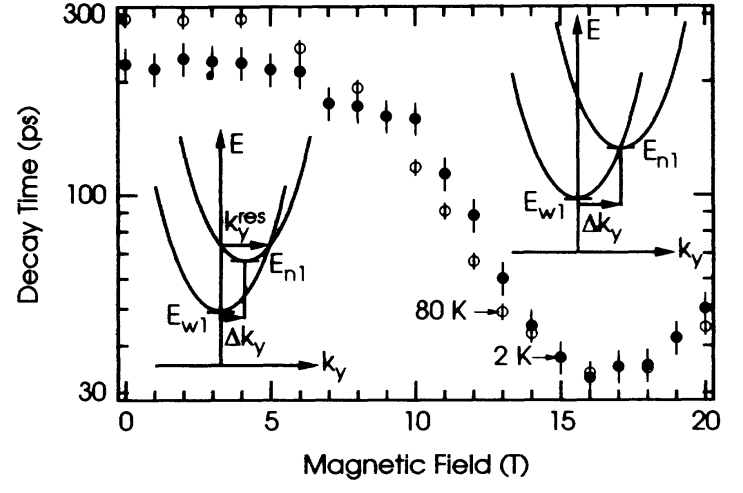

FIG. 2. Dependence of $\mathrm{QW} n$ luminescence decay time on magnetic field perpendicular to the growth direction for sample temperatures of $2 \mathrm{~K}$ and $80 \mathrm{~K}$. Insets: $k_{\nu}$ dispersion of the lowest electronic subbands $|w 1\rangle$ and $|n 1\rangle$ in an ADQW with magnetic field in the $x$ direction. The left diagram shows the situation where resonant transfer of electrons from $Q W_{n}$ to $\mathrm{QW}_{w}$ is not possible. In the right diagram resonant transfer is possible.

for the $z$ coordinate of $|w 1\rangle$ and $|n 1\rangle$. Semiclassically, $\Delta k_{y}$ is the momentum which an electron receives from the Lorentz force during the tunneling process. The left inset of Fig. 2 shows the resulting $k_{y}$ subband dispersion for an ADQW. The dispersion in $k_{x}$ is not influenced by the magnetic field and $k_{x}$ is therefore set to zero in the diagram. The $k_{y}$-dispersion curves of $|n 1\rangle$ and $|w 1\rangle$ intersect for any $B_{x} \neq 0$ at a wave vector $k_{y}^{\text {res }}\left(B_{x}\right)$. The intersection fulfills conservation of energy and transverse canonical momentum for resonant tunneling and therefore allows resonant transfer of electrons from $\mathrm{QW}_{n}$ to $\mathrm{QW}_{w}$ if the states at $k_{y}^{\text {res }}\left(B_{x}\right)$ are occupied. After excitation, electrons cool to a temperature of $50 \mathrm{~K}$ within the time resolution [17] and resonant transfer is therefore only possible if $k_{y}^{\text {res }}\left(B_{x}\right)$ is at the subband edge of $|n 1\rangle$ (right inset of Fig. 2). The condition for resonant transfer from $\mathrm{QW}_{n}$ to $\mathrm{QW}_{w}$ is therefore

$$
\Delta E=E_{n 1}-E_{w 1}=\frac{\left(\hbar \Delta k_{y}\right)^{2}}{2 m^{*}}=\frac{\left(e \Delta z B_{x}\right)^{2}}{2 m^{*}} .
$$

Equation (1) gives a resonance magnetic field of $15 \mathrm{~T}$ which is in reasonable agreement with the experiment. We do not observe resonant behavior in a magnetic field $B_{\|}$in the growth direction since resonant tunneling between different Landau levels is quantum mechanically forbidden in first order [5].

Resonant transfer from $\mathrm{QW}_{n}$ to $\mathrm{QW}_{w}$ is only observable if the electrons are scattered out of the resonant states in $\mathrm{QW}_{w}$. The resonant tunneling time is therefore determined by both the tunnel coupling through the barrier and an intersubband or intrasubband scattering process in $\mathrm{QW}_{\boldsymbol{w}}$. The influence of the subband distance $\Delta E$ on the tunneling times provides valuable information on the dominant scattering process. This possibility 
is unique for the present $B_{\perp}$ experiment which allows injection at finite in-plane wave vector and energy in the wide well and the possibility to tune these parameters by a simultaneous variation of the electric and the magnetic fields. We vary the subband distance $\Delta E$ by an electric field in the growth direction. Decreasing $\Delta E$ shifts the resonance to lower magnetic fields, as shown in Fig. 3. The solid line, calculated with Eq. (1), is in good agreement with the experiment. The small systematic deviation may be due to excitonic effects. The shift of the resonant magnetic field to lower values when $\Delta E$ is lowered proves that electrons tunnel; the resonant magnetic field for hole tunneling would increase with decreasing $\Delta E$. The dependence of the resonant decay times on $\Delta E$ is shown in Fig. 4. Resonant tunneling becomes fast if the subband spacing is above the GaAs longitudinal optical (LO) phonon energy of $\hbar \omega_{\mathrm{LO}}=36 \mathrm{meV}$, and the resonant decay times saturate for large $\Delta E$ at a minimum of $30 \mathrm{ps}$. This result shows that the transfer of electrons from $\mathrm{QW}_{n}$ to $\mathrm{QW}_{w}$ only becomes fast if they are rapidly swept out of the receiving state in $\mathrm{QW}_{w}$ by $\mathrm{LO}$ phonon emission.

The importance of LO phonon emission for resonant tunneling in a high magnetic field $B_{\perp}$ cannot be invoked to explain experiments with the same sample in the absence of a magnetic field where $|n 1\rangle$ and $|w 1\rangle$ were tuned into resonance by an electric field. A ground state resonance transfer time with a comparable value of $32 \mathrm{ps}$ was observed when $|n 1\rangle$ and $|w 1\rangle$ were slightly detuned to prevent backtunneling to the source quantum well [3]. The resonance had to be explained by defect assisted tunneling [18] since LO phonon participation was impossible [19]. Defect assisted transfer occurs under energy conservation but with momentum transfer in the range of (Bohr radius) ${ }^{-1}$, i.e., $10^{6} \mathrm{~cm}^{-1}$. The difference between the resonances is explained with the insets in Fig. 4: the labels $i$ and $f$ mark the positions of initial and final states for defect assisted scattering. Part (a) shows the identical dispersion of $|n 1\rangle$ and $|w 1\rangle$ when the resonance is

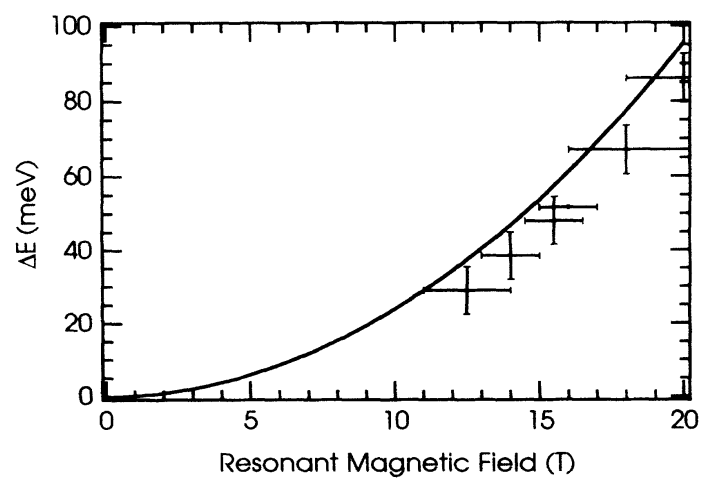

FIG. 3. Subband distance $\Delta E$ for various applied magnetic fields in resonance (bars) at $2 \mathrm{~K}$ in comparison to theory (curve). obtained by application of an electric field. Defect assisted transitions from the subband minimum of $|n 1\rangle$ to $|w 1\rangle$ with energy conservation but with small momentum change are possible. Part (b) shows the subband dispersion when resonance is obtained by application of a magnetic field. The possibility of a transition from a specific initial state at the minimum of $|n 1\rangle$ to a final state at $|w 1\rangle$ with energy conservation and small momentum transfer is strongly reduced. While in case (a) defect assisted transfer is dominant, in case (b) LO phonon assisted transfer prevails if $\Delta E \geq \hbar \omega_{\mathrm{LO}}$. The suppression of defect assisted transfer is a direct consequence of the particularity of magnetic field induced resonant tunneling, namely, that carriers can be injected in the receiving states at specific finite wave vector values and energies. The similar transfer times in a magnetically and an electrically tuned resonance are noteworthy since a different mechanism for loss of momentum in the receiving state must be involved; furthermore, in the second case the resonance is achieved for all in-plane wave vectors whereas in the first case the resonance is only obtained for one specific $k_{y}$. A possible explanation could be that in real samples well width fluctuations lead to inhomogeneous broadening of the states which slows down the resonance by 1 order of magnitude in the electrically obtained resonance. In the magnetic field case, inhomogeneous broadening only shifts the resonance to a slightly different $k_{y}$, which causes only a small influence on the transfer time. A detailed discussion of this behavior will be published elsewhere.

In conclusion, resonant tunneling between coupled quantum wells is investigated in a magnetic field perpendicular to growth direction. The transition from nonresonant to resonant tunneling reduces the tunneling time by a factor of 10 to a value of $30 \mathrm{ps}$. The resonance becomes particularly fast if $\mathrm{LO}$ phonon emission is possible in the tunneling process. Our results demonstrate that

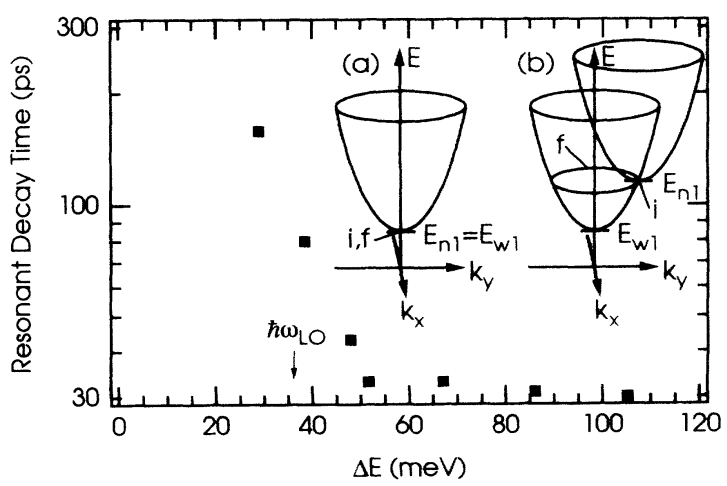

FIG. 4. Resonant luminescence decay time in dependence of the subband distance $\Delta E$ at $2 \mathrm{~K}$. Insets: In-plane dispersion of the subbands $|n 1\rangle$ and $|w 1\rangle$ when resonant tunneling is obtained by (a) an electric field in which case $|n 1\rangle$ and $|w 1\rangle$ lie on the same paraboloid (b) by a magnetic field. 
the direct observation of transfer times is essential for the interpretation of magnetic field induced resonances. Transfer with and without magnetic field ocurs with or without change of the in-plane motion of the carriers and the tunneling transfer mechanism can be completely different in both cases.

We would like to thank H.-J. Queisser, A. Fasolino, and J. Kuhl for valuable discussions and $\mathrm{H}$. Krath for technical assistance. The financial support of the Bundesministerium für Forschung und Technologie is gratefully acknowledged.

[1] E. Wolf, in Principles of Electron Tunneling Spectroscopy (Oxford University Press, New York, 1984).

[2] For a detailed recent review, see, e.g, A. C. Seabaugh, J. H. Luscombe, and J. N. Randall, Future Electron. Device J. 3, Suppl. 1, 9 (1993).

[3] A. P. Heberle, W. W. Rühle, M. G. W. Alexander, and K. Köhler, Semicond. Sci. Technol. 7, B421 (1992).

[4] D. Y. Oberli, J. Shah, T. C. Damen, J. M. Kuo, J. E. Henry, J. Lary, and S. M. Goodnick, Appl. Phys. Lett. 56, 1239 (1990).

[5] E. E. Mendez, L. Esaki, and W. I. Wang, Phys. Rev. B 33, 2893 (1986).

[6] M. L. Leadbeater, L. Eaves, P. E. Simmonds, G. A. Toombs, F. W. Sheard, P. A. Claxton, G. Hill, and M. A. Pate, Solid State Electron. 31, 707 (1988).

[7] J. Smoliner, W. Demmerle, G. Berthold, E. Gornik, G. Weimann, and W. Schlapp, Phys. Rev. Lett. 63, 2116 (1989).

[8] R. K. Hayden, D. K. Maude, L. Eaves, E. C. Valadares, M. Henini, F. W. Sheard, O. H. Hughes, J. C. Portal, and L. Cury, Phys. Rev. Lett. 66, 1749 (1991).
[9] Ulf Gennser, V. P. Kesan, D. A. Syphers, T. P. Smith III, S. S. Iyer, and E. S. Yang, Phys. Rev. Lett. 67, 3828 (1991).

[10] D. Y. Oberli, J. Shah, T. C. Damen, C. W. Tu, T. Y. Chang, D. A. B. Miller, J. E. Henry, R. F. Kopf, N. Sauer, and A. E. DiGiovanni, Phys. Rev. B 40, 3028 (1989).

[11] M. G. W. Alexander, M. Nido, W. W. Rühle, and K. Köhler, Phys. Rev. B 41, 12295 (1990).

[12] B. Deveaud, A. Chomette, F. Clerot, P. Auvray, A. Regreny, R. Ferreira, and G. Bastard, Phys. Rev. B 42, 7021 (1990).

[13] The slower transfer of the tunneling holes mainly leads to an increase of the rise time of the $\mathrm{QW}_{w}$ luminescence; see, e.g., M. Nido, M. G. W. Alexander, W. W. Rühle, T. Schweizer, and K. Köhler, Appl. Phys. Lett. 56, 355 (1990); Ph. Roussignol, A. Vinattieri, L. Carraresi, M. Colocci, and A. Fasolino, Phys. Rev. B 44, 8873 (1991).

[14] E. H. Hauge and J. A. Støvneng, Rev. Mod. Phys. 61, 917 (1989).

[15] J. Feldmann, G. Peter, E. O. Göbel, P. Dawson, K. Moore, C. Foxon, and R. J. Elliott, Phys. Rev. Lett. 59, 2337 (1987).

[16] T. Ando, J. Phys. Soc. Jpn. 39, 411 (1975).

[17] K. Leo, W. W. Rühle, and K. Ploog, Phys. Rev. B 38, 1947 (1988).

[18] G. Bastard, J. A. Brum, and R. Ferreira, in Electronic States in Semiconductor Heterostructures, Solid State Physics Vol. 44 (Academic Press, London, 1991), Chap. 12 , pp. 229-415.

[19] Transfer times for resonances with higher subbands where LO phonon emission becomes possible were in our samples similar to transfer times for ground state resonances if the coupling strength of the two wells, i.e., the integrated effective barrier height, was comparable (see Ref. [3]). This observation suggests that the dominant transfer mechanism is the same for all resonances. 